SLAC-PUB-9296

hep-ph/0207201

\title{
The Asymptotic Expansion of Lattice Loop Integrals Around the Continuum Limit
}

\author{
Thomas Becher and Kirill Melnikov \\ Stanford Linear Accelerator Center, Stanford University, Stanford, CA 94309, U.S.A.
}

\begin{abstract}
We present a method of computing any one-loop integral in lattice perturbation theory by systematically expanding around its continuum limit. At any order in the expansion in the lattice spacing, the result can be written as a sum of continuum loop integrals in analytic regularization and a few genuine lattice integrals ("master integrals"). These lattice master integrals are independent of external momenta and masses and can be computed numerically. At the one-loop level, there are four master integrals in a theory with only bosonic fields, seven in HQET and sixteen in QED or QCD with Wilson fermions.

PACS numbers: 11.15.Ha, 12.38.Bx
\end{abstract}

\section{INTRODUCTION}

Lattice regularization provides a non-perturbative formulation of quantum field theories and allows for the approximate numerical evaluation of the associated path integral [1]. Nevertheless, perturbative calculations with lattice regularization are often necessary, particularly when the problem at hand contains several disparate scales. In such situations it is sometimes possible to separate perturbative short distance physics from nonperturbative long distance physics. This separation depends on the regularization prescription, and in order to combine the perturbative results for the short distance part with the non-perturbative evaluation of the long distance matrix elements, both must be computed within the same regularization scheme. A typical example is the weak decay of a hadron. The physics associated with the high scale $M_{W}$ can be treated perturbatively by absorbing it into the Wilson coefficients of a low energy effective Lagrangian. The low energy physics is then obtained by evaluating the matrix elements of the operators that induce the decay. To match the lattice results for the matrix elements with the Wilson coefficients, typically obtained in dimensional regularization, a perturbative calculation in lattice regularization must be performed. Other important applications of lattice perturbation theory include the construction of actions with smaller discretization errors [2], and the extraction of quark masses [3] and the strong coupling constant [4, 5, 6] from lattice simulations.

It is well known that perturbative calculations with lattice regularization are difficult. The propagators and interaction vertices in lattice regularization are much more complicated than their continuum counterparts; lattice regularization also badly violates Lorentz invari- ance. Consequently, the standard set of tools for oneloop calculations in continuum perturbation theory does not appear to be useful in lattice calculations. In the standard approach to lattice perturbation theory the relevant loop integrals are therefore evaluated numerically, which has several drawbacks: a) the amount of numerical computations necessary for realistic calculations is huge; b) cancellations between individual diagrams can render numerical results unstable; c) the continuum limit, i.e. the limit in which the inverse lattice spacing becomes much larger than external momenta and masses, has to be taken numerically as well. There exist a number of techniques to both reduce the amount and enhance the precision of the numerical integrations involved. These methods rely on Reisz's power counting theorem [7] and use momentum subtractions to split the loop integrals into lattice tadpole contributions and a remainder whose continuum limit can be taken naively. The number of lattice tadpole-integrals is then reduced by exploiting relations among them [8, 9, 11] and techniques for the precise numerical evaluation of such integrals have been developed [1].

In this paper we demonstrate that a completely different approach to lattice perturbation theory is possible if advanced methods of continuum perturbation theory are applied to lattice calculations. We first show how to construct the systematic expansion of any lattice integral around its continuum limit by employing the technique of asymptotic expansions [12] developed for continuum loop integrals. After the expansion, the original lattice integral is expressed as a sum of two different contributions: continuum one-loop integrals that are regularized by means of analytic regularization and massless lattice tadpole-integrals. We then use integration-by-parts identities [13] to systematically reduce the number of tadpole- 
integrals to an absolute minimum. Those remaining are called master integrals and are evaluated numerically; their number depends on the theory and varies from four in any theory with only bosonic fields to sixteen in QED or QCD with Wilson fermions.

The advantage of this approach is that the one-loop lattice tadpole-integrals relevant for any conceivable calculation can be expressed in terms of master integrals in a process independent way. Therefore, once these relations are established and the master integrals are computed numerically, perturbation theory on the lattice reduces to perturbation theory in the continuum.

Our paper is organized as follows. After presenting our notation, we illustrate the method by applying it to massive tadpole-integrals. Although this example is very simple, it exhibits all the basic features of the method. We then study Feynman diagrams that involve static and Wilson fermions. In both cases the loop integrals can be expanded around their continuum limit in exactly the same way as the bosonic integrals. However, the comparatively more complicated form of the propagator for Wilson fermions leads to a larger number of master lattice integrals. Finally, we use the technique to rederive the known results for the gluon self-energy and the static and Wilson fermion two-point functions at one loop order in perturbation theory.

\section{PRELIMINARIES}

In addition to particle masses and momenta, the result of a calculation in lattice perturbation theory depends on the lattice spacing $a$. It is convenient to define dimensionless quantities by multiplying all momenta and masses by the lattice spacing so that $m=a m_{\text {phys }}, p=a p_{\text {phys }}$, etc. We use the standard lattice notation

$$
\widehat{p}^{2}=\sum_{\mu=1}^{d} \widehat{p}_{\mu}^{2}, \quad \widehat{p}_{\mu}=2 \sin \frac{p_{\mu}}{2},
$$

throughout the paper. The massive bosonic propagator on the lattice is

$$
G_{B}(k)=\frac{1}{\left(\widehat{k}^{2}+m^{2}\right)} .
$$

Loop integrals contain products of these propagators integrated over the Brillouin zone; a typical loop integral has the form

$$
\int_{-\pi}^{\pi} \frac{d^{d} k}{(2 \pi)^{d}} \frac{1}{\left(\widehat{k}^{2}+m^{2}\right)} \frac{1}{\left((\widehat{p+k})^{2}+m^{2}\right)},
$$

where $p$ is the external momentum and $d=4$ is the space-time dimension.

Our goal is to construct a procedure for expanding lattice integrals around their continuum limit. In terms of dimensionless quantities, this limit corresponds to all external momenta and masses of the particles becoming small. We begin by mapping the integration region in Eq.(3) to an infinite volume and define new integration variables $\eta_{\mu}$,

$$
\eta_{\mu}=\tan \left(k_{\mu} / 2\right) .
$$

In terms of the new variables, the loop integrations in Eq.(3) range from $-\infty$ to $+\infty$. It turns out to be convenient to introduce variables similar to Eq.(任) also for external momenta and to rescale $m$ to $\tilde{m}=m / 2$. The self-energy integral in Eq.(3) then becomes

$$
\begin{aligned}
& \frac{1}{4^{2} \pi^{d}} \int_{-\infty}^{\infty} \prod_{i=1}^{d} \frac{d \eta_{i}}{\left(1+\eta_{i}^{2}\right)}\left[\tilde{m}^{2}+\sum_{i=1}^{d} \frac{\eta_{i}^{2}}{\left(1+\eta_{i}^{2}\right)}\right]^{-1} \\
& \times\left[\tilde{m}^{2}+\sum_{i=1}^{d} \frac{\left(\rho_{i}+\eta_{i}\right)^{2}}{\left(1+\eta_{i}^{2}\right)\left(1+\rho_{i}^{2}\right)}\right]^{-1},
\end{aligned}
$$

where $\rho_{i}=\tan \left(p_{i} / 2\right)$.

The representation of lattice loop integrals as in Eq.(5) is similar in form to continuum loop integrals, albeit with unconventional propagators. The form of these propagators makes it impossible to apply the basic continuum techniques for performing loop calculations, such as Feynman parameterization and Passarino-Veltman reduction. However, we will show in the next section that more advanced techniques, such as recurrence relations and asymptotic expansions, are applicable.

\section{EXPANSION AROUND THE CONTINUUM LIMIT}

We demonstrate our method by evaluating the massive bosonic tadpole-integral. Although this is the simplest loop integral, the treatment of integrals with external momenta and different masses does not pose any additional difficulty; the part of these integrals that has nontrivial dependence on the masses and momenta, can be obtained by evaluating continuum one loop integrals. Using the variables defined above, the massive lattice tadpoleintegral can be written as

$$
G(\tilde{m})=\frac{1}{4 \pi^{d}} \int_{-\infty}^{\infty} \prod_{i=1}^{d} \frac{d \eta_{i}}{\left(1+\eta_{i}^{2}\right)}\left[\tilde{m}^{2}+D_{B}(\eta)\right]^{-1},
$$

where

$$
D_{B}(\eta)=\sum_{i=1}^{d} \frac{\left(\eta_{i}\right)^{2}}{\left(1+\eta_{i}^{2}\right)} .
$$

We are interested in the continuum limit of Eq.(6), $\tilde{m} \rightarrow 0 . G(\tilde{m})$ therefore depends on a small parameter and this fact can be used to simplify the calculation of the integral in Eq.(6). Unfortunately, a straightforward Taylor expansion of the integrand in $\tilde{m}$ is not possible, since $G(\tilde{m})$ is not an analytic function of $\tilde{m}$. 
Traditionally, lattice loop integrals with external momenta are simplified using Reisz's power counting theorem [7]. In this approach, a number of momentum subtractions are performed on the integral. These subtractions split the integral into two parts: a polynomial in the external momenta with tadpole-integrals as coefficients, and a remainder that depends non-trivially on the external momenta, but whose continuum limit can be taken naively. When applied to theories with massless fields, this method produces infrared divergent tadpole-integrals, and makes it necessary to introduce an infrared regulator at intermediate stages of the calculation. These infrared divergences illustrate that the loop integrals are not analytic functions of the small momenta and masses; this is reflected in the appearance of terms such as $\log (\tilde{m})$.

As is well known from continuum perturbation theory, the fact that the Taylor expansion of the Feynman integral in a small parameter does not commute with the loop integration is not an obstacle for constructing a highly practical procedure to perform the expansion before the integration; this technique is known as the asymptotic expansion of loop integrals 12. To apply this procedure to lattice loop integrals we must introduce an additional regulator into the integral in Eq.(6). We use analytic regularization, and replace the integral $G(\tilde{m})$ by

$$
G(\tilde{m})=\frac{1}{4 \pi^{d}} \int_{-\infty}^{\infty} \prod_{i=1}^{d} \frac{d \eta_{i}}{\left(1+\eta_{i}^{2}\right)}\left[\tilde{m}^{2}+D_{B}(\eta)\right]^{-1-\delta} .
$$

After the regulator is introduced, the expansion of $G(\tilde{m})$ in $\tilde{m}$ can be constructed; the limit $\delta \rightarrow 0$ can be taken at the end. The result for $G(\tilde{m})$ is obtained as a sum of two contributions:

$$
G(\tilde{m})=G_{\text {soft }}(\tilde{m})+G_{\text {hard }}(\tilde{m}) .
$$

The soft and hard contributions are calculated by applying the following procedure to the integrand in Eq.(8):

- Soft: assume that all the components of the loop momentum $\eta$ are small, $\eta_{i} \sim \tilde{m} \ll 1$. Perform the Taylor expansion of the integrand in Eq.(8) in the small quantities $\eta_{i}$ and $\tilde{m}$. The expansion coefficients in this region are standard continuum one loop integrals, regularized analytically. Note that no restriction on the integration region is introduced.

- Hard: assume that all the components of the loop momentum are large, $\eta_{i} \sim 1 \gg \tilde{m}$ and Taylor expand the integrand in $\tilde{m}$. The expansion coefficients are given by the functions $H\left(\left\{a_{i}\right\} ; n\right)$, defined in Eq.(13) below. These functions are related by algebraic and integration-by-parts identities.

We note that the two regions discussed above do not account for all the possible scalings of the components of the loop momentum. A potential contribution arises from regions where some components of the loop momentum are soft and the other are hard. Following the above logic, the integrand of Eq.(8) should be expanded in the small quantities; it is easy to see that in this case the analytic regularization as introduced in Eq.(8) does not fully regulate the resulting expressions. The contributions of these mixed regions are set to zero. To justify this prescription we consider another possible choice of the regulator. We regulate the loop integrals by including a factor $\left(\sin ^{2}\left(k_{i} / 2\right)\right)^{\epsilon}$ in the measure for each component of the loop momenta. The disadvantage of this regulator is that the resulting soft parts are more difficult to calculate than in analytic regularization. Its advantage is that it fully regulates the mixed regions, where some loop momentum components are small and others are large. The integrals occurring in the mixed region are scaleless and therefore vanish. We now introduce both the sine-function and the analytic regulator simultaneously to show that the mixed regions do not contribute. Since the sine-regulator does not contain any unregulated regions, the original integral is recovered by the prescription

$$
G(M)=\lim _{\delta \rightarrow 0} \lim _{\epsilon \rightarrow 0} G(M, \epsilon, \delta) .
$$

In this prescription the mixed regions do not contribute. After showing that for non-zero $\delta$ both the hard and the soft parts are analytic functions of $\epsilon$ at $\epsilon=0$, we can take the limit $\epsilon \rightarrow 0$ at the level of the integrand and thus recover the integrals in analytic regularization. To prove analyticity of the hard part, we use the relations between the integrals that appear in the hard part to express them through convergent master integrals and verify that there are no $1 / \epsilon$ singularities in the relations of any hard integral to the master integrals. We can therefore drop the sine-function regulator and ignore the contributions of the mixed regions. The analytically regularized integral is then recovered and its expansion is constructed according to the rules given after Eq.(9). We now discuss how the soft and the hard contributions to the massive tadpole-integral are calculated.

\section{A. Soft part of the integrals}

The soft part of $G(\tilde{m})$ is easy to obtain. After expanding the integrand, we arrive at analytically regularized continuum one loop integrals. Higher orders in the expansion introduce a large number of strongly divergent integrals with non-covariant numerators; a consequence of broken Lorentz invariance on the lattice. This is not an obstacle, since all such integrals can be easily evaluated using the identity

$$
\begin{aligned}
& \int \mathrm{d}^{d} \eta \frac{1}{\left(\eta^{2}+\tilde{m}^{2}\right)^{\alpha}} \prod_{i=1}^{d}\left(\eta_{i}^{2}\right)^{a_{i}}=(\tilde{m})^{d-2 \alpha+2 \sum_{i} a_{i}} \\
& \times \frac{\Gamma\left(\alpha-d / 2-\sum_{i} a_{i}\right)}{\Gamma(\alpha)} \prod_{i} \Gamma\left(\frac{1}{2}+a_{i}\right) .
\end{aligned}
$$


The calculation of the soft part of integrals that depend on external momenta or several distinct masses is equally simple: this part is always given by continuum integrals in analytic regularization and can be brought to the form of Eq.(11) using standard methods, such as Feynman parameters.

The result for the soft part of the massive tadpoleintegral is

$$
\begin{aligned}
4 \pi^{2} G_{\text {soft }}(\tilde{m}) & =-\tilde{m}^{2}\left(\frac{1}{\delta}+1-2 \log (\tilde{m})\right) \\
+ & \tilde{m}^{4}\left(\frac{1}{2 \delta}+\frac{3}{4}-\log (\tilde{m})\right)+O\left(\tilde{m}^{6}\right) .
\end{aligned}
$$

\section{B. Hard part of the integrals}

We now consider the hard contribution to $G(\tilde{m})$. Recall that the hard part is obtained by expanding Eq.(8) in a Taylor series in $\tilde{m}$; the resulting expression is

$$
\begin{aligned}
& 4 \pi^{4} G(\tilde{m})_{\text {hard }}=H(\{1,1,1,1\}, 1) \\
& \quad-\tilde{m}^{2}(1+\delta) H(\{1,1,1,1\}, 2) \\
& +\frac{\tilde{m}^{4}}{2}(1+\delta)(2+\delta) H(\{1,1,1,1\}, 3)+\ldots .
\end{aligned}
$$

The functions $H\left(\left\{a_{i}\right\} ; n\right)$ are defined by

$$
H\left(\left\{a_{i}\right\} ; n\right)=\int_{-\infty}^{\infty} \prod_{i=1}^{d} \frac{d \eta_{i}}{\left(1+\eta_{i}^{2}\right)^{a_{i}}}\left[D_{B}(\eta)\right]^{-n-\delta}
$$

where $a_{i}$ and $n$ are integers. In a theory with only bosonic propagators, the functions $H\left(\left\{a_{i}\right\} ; n\right)$ form the full set of genuine lattice integrals needed to perform one-loop calculations in lattice perturbation theory. We are forced to consider $H$ integrals with $a_{i} \neq 1$ for two reasons: they appear in the expansion of loop integrals with external momenta and/or nontrivial numerators [14; in addition, they are needed in the reduction process of an arbitrary $H$-integral to the master integrals.

We now use integration-by-parts identities to fully exploit the algebraic relations between the various integrals $H\left(\left\{a_{i}\right\}, n\right)$. These relations are derived using the fact that the integral of a total derivative vanishes in analytic regularization:

$$
0=\int_{-\infty}^{\infty} d^{d} \eta \frac{\partial}{\partial \eta_{\mu}}\left\{\eta_{\mu} \prod_{i=1}^{d} \frac{1}{\left(1+\eta_{i}^{2}\right)^{a_{i}}}\left[D_{B}(\eta)\right]^{-n-\delta}\right\}
$$

for each value of $\mu=1,2, \ldots d$. Computing the derivative in Eq.(14) and rewriting the resulting expression in terms of the functions $H\left(\left\{a_{i}\right\}, n\right)$, we obtain an algebraic relation between integrals with different values of $\left\{a_{i}\right\}$ and $n$. Another equation can be obtained by partial fractioning, i.e. by using the linear dependence of the five "propagators" in the function $H\left(\left\{a_{i}\right\}, n\right)$. The complete set of algebraic relations is therefore

$$
\begin{aligned}
& 0=\left\{\boldsymbol{n}^{-}+\sum_{i=1}^{d}\left(\boldsymbol{a}_{i}^{+}-1\right)\right\} H \\
& 0=\left\{1+2 a_{i}\left(\boldsymbol{a}_{i}^{+}-1\right)+2(n+\delta) \boldsymbol{n}^{+} \boldsymbol{a}_{i}^{+}\left(\boldsymbol{a}_{i}^{+}-1\right)\right\} H .
\end{aligned}
$$

The conventions are such that the operator $\boldsymbol{a}_{i}^{ \pm}$increases (decreases) the index $a_{i}$ by one.

Similar integration-by-parts relations for lattice integrals were first studied in 8, 9, where it was shown that the entire class of integrals $H\left(\left\{a_{k}\right\} ; n\right)$ can be reduced to $d$ master integrals in $d$ dimensions. Here, we neither attempt to solve these equations explicitly nor to rewrite them in such a form that the reduction of a given index is manifest. Instead, we adopt a brute force strategy and use computer algebra to explicitly solve the equations for a given range of indices. An efficient algorithm for solving such recurrence relations has been described in 15. First, a criterion which selects a simpler integral out of any two integrals is chosen. Typically, integrals with lower values of the indices are considered to be simpler. The above equations are then solved for a very limited range of indices, using Gauss's elimination method. The calculation is repeated after supplementing the chosen set of equations with a few relations involving higher index values. By iterating this procedure, the equations (15) can be solved for the entire index range needed in a given calculation. The advantage of this brute force method is that it immediately generalizes to integrals involving more complicated propagators (e.g. those of Wilson fermions) or to higher loops. In the continuum, this strategy has lead to the solution of problems that would have been very difficult to tackle by manipulating recurrence relations by hand, for example the four point functions of QCD at two loops [16.

Having established that the bosonic case requires four master integrals, we can choose those in any way we like. It is convenient to choose master integrals that are convergent in the limit $\delta \rightarrow 0$, since their expansion in $\delta$ can be obtained by expanding the integrands in Eq.(13) in a power series in $\delta$. A possible choice that satisfies this criterion is $H_{0}=H(\{1,1,1,1\} ; 1), H_{1}=H(\{1,1,1,2\} ; 1)$, $H_{2}=H(\{1,1,2,2\} ; 1), H_{3}=H(\{1,2,2,2\} ; 1)$.

It is a fairly easy task to numerically evaluate the finite bosonic integrals to relatively high precision. In the case of $H$ integrals, this task can be further simplified by using the Schwinger representation for the propagators, which permits a reduction to one-dimensional integrals. Writing

$$
\frac{1}{\left(\widehat{k}^{2}\right)^{\alpha}}=\frac{1}{\Gamma(\alpha)} \int_{0}^{\infty} t^{\alpha-1} e^{-t \widehat{k}^{2}}
$$

the integration over the momentum $k$ factorizes and can be done analytically, leaving one dimensional integrals 
over products of modified Bessel functions $I_{n}(t / 2)$ for numerical evaluation. In this way, we obtain the following one-dimensional integral representations for the master integrals $H_{0-3}$ defined above:

$$
\begin{array}{r}
H_{i}=\frac{\pi^{4}}{2^{i}} \int_{0}^{\infty} d t e^{-2 t} I_{0}(t / 2)^{4-i}\left\{I_{0}(t / 2)+I_{1}(t / 2)\right\}^{i} \\
\times\left[1+\left(\gamma_{E}+\ln t\right) \delta\right]+O\left(\delta^{2}\right) .
\end{array}
$$

The precise numerical evaluation of these one dimensional integrals is straightforward. Our results for the master integrals are

$$
\begin{aligned}
& H_{0}=60.3676829-16.7691976 \delta+\mathcal{O}\left(\delta^{2}\right), \\
& H_{1}=36.0154101-1.83118998 \delta+\mathcal{O}\left(\delta^{2}\right), \\
& H_{2}=22.1620171+4.75278418 \delta+\mathcal{O}\left(\delta^{2}\right), \\
& H_{3}=14.1541117+7.32383339 \delta+\mathcal{O}\left(\delta^{2}\right) .
\end{aligned}
$$

Since it is rather simple to obtain the above results, the integrals $H_{0-3}$ form a convenient basis. However, in order to facilitate the comparison of our results with the literature, we point out that other choices of the master integrals are possible. One possibility is

$$
\begin{aligned}
& \frac{1}{\pi^{2}} H(\{1,1,1,1\} ; 0)=\pi^{2}, \\
& \frac{1}{\pi^{2}} H(\{1,1,1,1\} ; 1)=4 \pi^{2} b_{1}, \\
& \frac{1}{\pi^{2}} H(\{1,1,1,1\} ; 2)=-\frac{1}{\delta}-2 \ln 2+1+16 \pi^{2} b_{2}, \\
& \frac{1}{\pi^{2}} H(\{1,1,1,1\} ; 3)=-\frac{1}{2 \delta}-\ln 2+\frac{3}{4}+64 \pi^{2} b_{3} .
\end{aligned}
$$

The three constants $b_{1,2,3}$ are closer to traditional choices of the basis numbers. For instance, Ref. [9] uses the notation $Z_{0}=b_{1}, F_{0}=16 \pi^{2} b_{2}+\gamma_{E}$ and $12 \pi^{2} Z_{1}=$ $26+3 \pi^{2}-384 \pi^{2} b_{2}+3072 \pi^{2} b_{3}$. The two constants $b_{1}$ and $b_{2}$ are equal to $P_{1}$ and $P_{2}$ of [11]; however, $b_{3} \neq P_{3}$. We can use the relations between the two sets of basis integrals to obtain the constants $b_{1-3}$. For example, the integral $H(1,1,1,1,2)$ is given by

$$
\begin{aligned}
H(1,1,1,1,2) & =\frac{(3 \delta+5)}{12(1+\delta)} H_{0}+\frac{(3+2 \delta)}{6(1+\delta)} H_{1} \\
& +\frac{\left(2 \delta+\delta^{2}-6\right)}{2(1+\delta) \delta} H_{2}+\frac{(2-\delta)^{2}}{(1+\delta) \delta} H_{3}
\end{aligned}
$$

Using the numerical results for the integrals $H_{0-3}$ presented above, we then obtain the value of $b_{2}$. Performing similar calculations for the other integrals, we arrive at

$$
\begin{aligned}
& b_{1}=0.15493339 \\
& b_{2}=0.02401318 \\
& b_{3}=0.00158857
\end{aligned}
$$

Finally, we present the result for the massive lattice tadpole-integral by combining the soft and the hard contributions discussed above. In Fig. 11 we compare the numerical evaluation of $G(m)$ with the expansion around its continuum limit, including terms up to $O\left(m^{16}\right)$. We note that the expansion converges up to relatively large values of the mass, $m \sim 2.5$, where the Compton wavelength of the particle is less than half the lattice spacing.

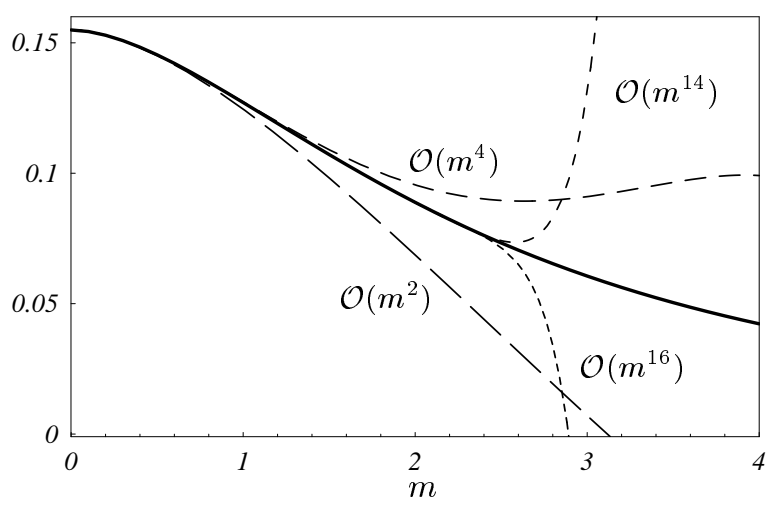

FIG. 1: Comparison of the numerical evaluation of the propagator at the origin $G(m)$ with the expansion around its continuum limit. The thick line corresponds to the numerical result while the four dashed lines show the expansions in $m=m_{\text {phys }} a$ to the indicated orders. Recall that $m=2 \tilde{m}$.

\section{OTHER PROPAGATORS}

The scalar lattice propagator in Eq.(2) arises in the simplest of infinitely many possible discretizations of the continuum action. Unfortunately, this simplest choice leads to large discretization errors. These can be reduced by introducing improved actions which include higher dimensional operators chosen to cancel lattice artifacts to a given order in the expansion around the continuum limit 2]. In doing so, the free propagator usually gets modified. A famous example is provided by fermions on the lattice, where the simplest discretization leads to the well known doubling problem. Among the many solutions to this problem, the most common is to follow Wilson's suggestion and add the dimension-five term $\bar{\psi} \square \psi$ to the fermion action [10].

Despite the fact that the inclusion of higher dimensional operators in more refined versions of the lattice action changes the propagator, our strategy remains the same. The structure of the soft part of the loop integrals remains unchanged, since it encodes the behavior at small momenta; the hard part, however, becomes a function of the couplings of the higher dimensional operators. This typically leads to more complicated recurrence relations for the hard integrals, but the approach described in the previous section is robust enough to solve the resulting recurrence relations. We now illustrate this for both static and Wilson fermions. 


\section{A. Static Fermions}

In a bound state of a heavy $\left(m_{q} \gg \Lambda_{Q C D}\right)$ and a light quark, the heavy quark remains almost static, and the probability of finding virtual heavy quark-antiquark pairs is small. In this situation the heavy quark can be treated as an approximately static color source. The systematic expansion around the static limit is obtained using the Heavy Quark Effective Theory (HQET) 17. This effective theory provides a method of putting heavy quarks on the lattice that does not require excessively small lattice spacings.

The discretized heavy quark propagator is 18

$$
G_{\text {static }}\left(k_{4}\right)=\frac{1}{i\left(1-e^{i k_{4}}\right)+i \epsilon} .
$$

In the static limit the pole in the propagator occurs at $k_{4}=0$, rendering an $i \epsilon$ prescription necessary even when working in Euclidean space. Using the variables introduced in Section II, the static propagator can be written as

$$
G_{\text {static }}\left(\eta_{4}\right)=\frac{1-i \eta_{4}}{2 \eta_{4}+i \epsilon} .
$$

The soft integrals are obviously the ordinary continuum HQET integrals. After regulating the bosonic propagator, the hard integrals become

$$
H\left(\left\{a_{i}\right\} ; n, m\right)=\int_{-\infty}^{\infty} \prod_{i=1}^{d} \frac{d \eta_{i}}{\left(1+\eta_{i}^{2}\right)^{a_{i}}} \frac{\left[D_{B}(\eta)\right]^{-n-\delta}}{\left(\eta_{4}+i \epsilon\right)^{m}} .
$$

Again, we wish to use integration-by-parts relations to reduce the number of independent hard integrals to an absolute minimum. Both the relations obtained by differentiation with respect to $\eta_{1-3}$ and the partial fractioning identity for the bosonic propagator coincide with the relations given in the previous section. The integrationby-parts identity obtained by differentiating with respect to $\eta_{4}$ becomes

$$
\begin{aligned}
& \left\{2(n+\delta)\left(\boldsymbol{a}_{1}^{+}-\boldsymbol{m}^{++}\right) \boldsymbol{a}_{1}^{+} \boldsymbol{n}^{+}\right. \\
& \left.-\left[(3+m) \boldsymbol{m}^{++}+2 a_{1} \boldsymbol{a}_{1}^{+}\right] \boldsymbol{m}^{++}\right\} H=0 .
\end{aligned}
$$

The partial fractioning identity for the static propagator is

$$
\left\{\left(1-\boldsymbol{a}_{1}^{+}\right) \boldsymbol{m}^{++}-\boldsymbol{a}_{1}^{+}\right\} H=0 .
$$

An additional algebraic identity is obtained by replacing

$$
\frac{1}{\eta_{4}+i \epsilon}=\mathcal{P} \frac{1}{\eta_{4}}-i \pi \delta\left(\eta_{4}\right) .
$$

The principal value contribution to $H\left(\left\{a_{i}\right\} ; n, 1\right)$ vanishes because of the symmetry $\eta_{4} \rightarrow-\eta_{4}$, and the contribution from the $\delta$-function is independent of $a_{4}$ :

$$
H\left(\left\{a_{1}, a_{2}, a_{3}, a_{4}\right\} ; n, 1\right)=H\left(\left\{a_{1}, a_{2}, a_{3}, 0\right\} ; n, 1\right) .
$$

Performing the loop integration over $\eta_{4}$, it is easy to see that the integrals $H\left(\left\{a_{1}, a_{2}, a_{3}, 0\right\} ; n, 1\right)$ reduce to the bosonic hard integrals in three dimensions. The lattice HQET master integrals are therefore given by the four bosonic master integrals discussed in the previous section, and the three bosonic master integrals in $d=3[19$. For the three-dimensional bosonic integrals we choose the basis

$$
\begin{aligned}
\frac{1}{\pi^{3}} H(\{1,1,1\}, 0) & =1+\mathcal{O}(\delta), \\
\frac{1}{4 \pi^{3}} H(\{1,1,1\}, 1) & =s_{1}+\mathcal{O}(\delta) \approx 0.252731010, \\
\frac{1}{16 \pi^{3}} H(\{1,1,1\}, 2) & =s_{2}+\mathcal{O}(\delta) \approx 0.012164159 .
\end{aligned}
$$

\section{B. Wilson fermions}

Wilson fermions 10 are often used to introduce fermions on the lattice, and we therefore consider this case in detail. The propagator of a Wilson fermion is

$$
G(k)=\frac{-i \sum_{\mu} \gamma_{\mu} \sin \left(k_{\mu}\right)+m+\frac{r}{2} \widehat{k}^{2}}{4 D_{F}(k, m)} .
$$

For our discussion only the form of its denominator,

$$
4 D_{F}(k, m)=\sum_{\mu} \sin ^{2}\left(k_{\mu}\right)+\left(m+\frac{r}{2} \widehat{k}^{2}\right)^{2},
$$

is relevant. In the continuum limit, external momenta and masses tend to zero while the Wilson parameter $r \neq 0$ stays fixed. In the notation of Section II the denominator is

$$
D_{F}(\eta, \tilde{m})=\sum_{\mu} \frac{\eta_{\mu}^{2}}{\left(1+\eta_{\mu}^{2}\right)^{2}}+\left(\tilde{m}+r D_{B}(\eta)\right)^{2} .
$$

We consider a general one loop integral with both bosonic and fermionic propagators and regulate the fermionic propagator. The integrals that appear in the hard part of the expansion are

$$
\begin{aligned}
& H \equiv H\left(\left\{a_{i}\right\}, n, m\right)= \\
& \int \mathrm{d}^{d} \eta \prod_{i=1}^{d} \frac{1}{\left(1+\eta_{i}^{2}\right)^{a_{i}}} D_{B}(\eta)^{-n} D_{F}(\eta, 0)^{-m-\delta} .
\end{aligned}
$$

We again begin by writing down the algebraic and integration-by-parts identities for the hard integrals. We find three identities by partial fractioning:

$$
\begin{aligned}
& \left\{1+\sum_{i}\left(\boldsymbol{a}_{i}^{+}-1\right) \boldsymbol{n}^{+}\right\} H=0, \\
& \left\{1+\sum_{i}\left(\boldsymbol{a}_{i}^{+}-1\right) \boldsymbol{m}^{+}\right. \\
& \left.\quad-\left(r^{2}-1\right)\left[\sum_{i}\left(\boldsymbol{a}_{i}^{+}-1\right)\right]^{2} \boldsymbol{m}^{+}\right\} H=0, \\
& \left\{\left[\boldsymbol{m}^{-}+\sum_{i} \boldsymbol{a}_{i}^{+}\left(\boldsymbol{a}_{i}^{+}-1\right)\right] \boldsymbol{n}^{++}-r^{2}\right\} H=0
\end{aligned}
$$


$d$ additional ones arise from integration-by-parts:

$$
\begin{gathered}
\left\{1+2(m+\delta) \boldsymbol{a}_{i}^{+}\left(\boldsymbol{a}_{i}^{+}-1\right) \boldsymbol{m}^{+}\left[2 \boldsymbol{a}_{i}^{+}-1-2 r^{2} \sum_{j}\left(\boldsymbol{a}_{j}^{+}-1\right)\right]\right. \\
\left.+2 a_{i}\left(\boldsymbol{a}_{i}^{+}-1\right)+2 n \boldsymbol{a}_{i}^{+}\left(\boldsymbol{a}_{i}^{+}-1\right) \boldsymbol{n}^{+}\right\} H=0 .
\end{gathered}
$$

These equations are considerably more complicated than the ones encountered in the scalar case. Not only does the index space become six-dimensional, but the recurrence relations also shift indices by up to three units. While the solution of the bosonic recursion relations in the index range needed for the applications of Section V was accomplished within hours, about a day of computer time was required to perform the reduction in the fermion case for $r=1$. We are left with sixteen master integrals, which must be computed numerically. For purely fermionic integrals this number reduces to ten.

Because of the complicated structure of the fermion denominator, we cannot use the Schwinger representation (16) to arrive at one-dimensional representations for the master integrals. A general strategy to obtain accurate results for more complicated cases is to choose a basis of integrals that are very convergent in the infrared, such as those with negative powers of propagators. Because of the simple behavior of the integrand at small values of $k$, these integrals can be obtained by replacing the integration with a sum over a small lattice. We illustrate this point for the purely bosonic integrals. Instead of the four integrals $H_{i}$ defined in Eq. (17), we could have chosen to evaluate four integrals from the set

$$
H(\{1,1,1,1\},-n)=\int_{-\pi}^{\pi} d^{4} k\left(\frac{\widehat{k}^{2}}{4}\right)^{n+\delta}
$$

to second order in $\delta$. Replacing $k_{i} \rightarrow \frac{2 \pi}{L}\left(l_{i}-\frac{1}{2}\right)$ and trading the integral over the Brillouin zone for a sum over $l_{i}=1, \ldots, L$, we obtain the value of the above integral up to finite size effects of order $1 / L^{4+2 n}$. Even the choice $n=0,1,2,3$ gives rather accurate values for lattice sizes $L>50$. In reference [9], this strategy was applied to fermionic tadpole-integrals and precise values for the corresponding master integrals were derived. We have verified the first few digits of their numerical results.

\section{APPLICATIONS}

To test this method in a practical application, we use it to rederive the known results for the gluon and fermion self-energies in QCD. The calculation of these quantities is instructive since it exhibits all the complications that arise in lattice perturbation theory. For example, the expansion of the gauge action around the continuum limit leads to infinitely many vertices. When they multiply divergent integrals, sub-leading vertices contribute in the continuum limit and lattice calculations consequently involve a larger number of graphs, than the corresponding calculations in the continuum theory. Furthermore, the vertices in lattice gauge theory have a complicated form. It takes roughly one page to write down the Feynman rule for the four gluon vertex [1]. The tensor structure of the graphs can therefore be quite lengthy. Summing over the internal indices in the first graph in Fig. 22 one ends up with several hundred terms. However, the large number of terms is not a significant obstacle since the technique described here can be easily automated.

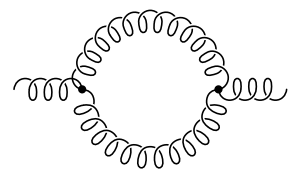

(a)

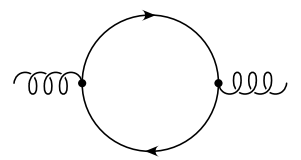

(c)

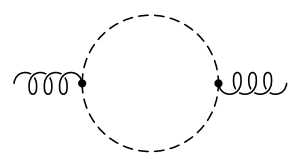

(e)

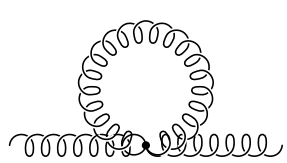

(b)

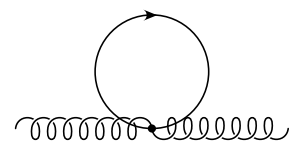

(d)

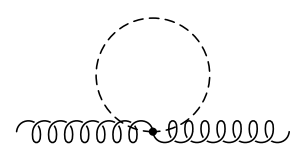

(f) rovorosoxponoded

(g)

FIG. 2: Feynman diagrams contributing to the gluon self energy at $O\left(g^{2}\right)$. The graphs $d, f$ and $g$ have no continuum analog. The graph $g$ arises from the expansion of the Haar measure.

\section{A. Gluon self-energy}

The individual gluon self-energy graphs of Fig. 2 diverge quadratically with the lattice spacing, and we have therefore expanded them to sub-leading order. They contain terms proportional to the the lattice invariants $g_{\mu \nu}$, $p_{\mu} p_{\nu}$ and $p_{\mu}^{2} g_{\mu \nu}$. In the sum the quadratic divergences and the non-covariant pieces cancel and the result for the inverse gluon propagator becomes

$$
\begin{array}{r}
\Gamma_{\mu \nu, a b}^{G}(p)=\left(g_{\mu, \nu}-p_{\mu} p_{\nu}\right) \delta_{a b}\left[1+\frac{g^{2}}{(4 \pi)^{2}}\left(\Gamma_{\text {hard }}\right.\right. \\
\left.\left.+\Gamma_{\text {soft }}\right)\right]+(\xi+1) \delta_{a b} p_{\mu} p_{\nu}
\end{array}
$$


The gluonic contribution to the self-energy is

$$
\begin{gathered}
\Gamma_{\mathrm{soft}}^{(G)}=N\left\{-\frac{5}{3 \delta}-\frac{14}{9}-\frac{\xi^{2}}{4}+\frac{5}{3} \ln \frac{p^{2}}{4}\right. \\
\left.+\xi\left(\frac{1}{2 \delta}-\frac{5}{6}-\frac{1}{2} \ln \frac{p^{2}}{4}\right)\right\}, \\
\Gamma_{\text {hard }}^{(G)}=N\left\{\frac{5}{3 \delta}-\frac{14}{9}-\pi^{2}+\frac{2 \pi^{2}}{N^{2}}-\frac{14 \pi^{2}}{9} b_{1}-\frac{80 \pi^{2}}{3} b_{2}\right. \\
\left.+\frac{5}{3} \ln 4+\xi\left(-\frac{1}{2 \delta}-\frac{1}{6}-\frac{\pi^{2}}{3} b_{1}+8 \pi^{2} b_{2}-\ln 2\right)\right\},
\end{gathered}
$$

where $N=3$ is the number of colors. The infrared divergence at $\delta=0$ in the hard part cancels the ultraviolet divergence of the soft part after the two terms are added. For $r=1$, the Wilson fermion contribution to the gluon vacuum polarization is

$$
\begin{aligned}
& \Gamma_{\text {hard }}^{(f)}=\frac{1}{2}\left\{\frac{4}{3 \delta}-1.376\right\} \\
& \Gamma_{\text {soft }}^{(f)}=\frac{1}{2}\left\{-\frac{4}{3} L_{\delta}-\frac{10}{9}+\frac{16 x}{3}+\frac{4}{3}(1-2 x) f(x)\right\},
\end{aligned}
$$

where $x=m^{2} / p^{2}, L_{\delta}=1 / \delta-\ln \left(m^{2} / 4\right)$, and

$$
f(x)=\sqrt{1+4 x} \ln \frac{\sqrt{1+4 x}+1}{\sqrt{1+4 x}-1} .
$$

After combining the hard and soft parts, we reproduce the result of Refs. [20, 21].

\section{B. Wilson fermion self-energy}

For $r=1$, the self-energy of a Wilson fermion with mass $m$ is

$$
\Sigma(p)=\frac{C_{F} g^{2}}{(4 \pi)^{2}}\left(\Sigma^{(0)}+i \not p \Sigma^{(1)}+m \Sigma^{(2)}\right) .
$$

The hard part is given by

$$
\begin{aligned}
& \Sigma_{\text {hard }}^{(0)}=-51.435, \\
& \Sigma_{\text {hard }}^{(1)}=\frac{1+\xi}{\delta}+13.74-2.91 \xi, \\
& \Sigma_{\text {hard }}^{(2)}=\frac{4+\xi}{\delta}+3.45-3.41 \xi ;
\end{aligned}
$$

the soft part is

$$
\begin{aligned}
& \Sigma_{\mathrm{soft}}^{(0)}=0 \\
& \Sigma_{\mathrm{soft}}^{(1)}=(1+\xi)\left\{-L_{\delta}+x-\frac{1}{2}+\left(1-x^{2}\right) \ln \frac{1+x}{x}\right\}, \\
& \Sigma_{\mathrm{soft}}^{(2)}=(4+\xi)\left\{-L_{\delta}-1+(1+x) \ln \frac{1+x}{x}\right\} .
\end{aligned}
$$

with $x=m^{2} / p^{2}, L_{\delta}=1 / \delta-\ln \left(m^{2} / 4\right)$.

\section{Static quark self-energy}

The wave function renormalization of the heavy quark is infrared divergent. Regulating the infrared divergence with a gluon mass $\lambda$, the self-energy takes the form

$$
\Sigma_{\mathrm{HQET}}(\omega)=\frac{C_{F} g^{2}}{(4 \pi)^{2}}\left(\Sigma_{\mathrm{hard}}+\Sigma_{\mathrm{soft}}\right),
$$

where $\left(x=\lambda^{2} / \omega^{2}, L_{\delta}=1 / \delta-\ln \left(\lambda^{2} / 4\right)\right)$ and

$$
\begin{aligned}
\Sigma_{\text {hard }}= & -8 i \pi^{2} s_{1}+\omega\left\{-\frac{(2-\xi)}{\delta}+8 \pi^{2} s_{1}-2 b_{1} \pi^{2}\right. \\
& \left.+16 b_{2} \pi^{2}(2-\xi)-\xi-2(2-\xi) \ln 2\right\}, \\
\Sigma_{\text {soft }}= & \omega\left\{(2-\xi) L_{\delta}+4+\xi\right. \\
& \left.+\frac{(2 x+(2-\xi))}{\sqrt{1+x}}\left(i \pi+\ln \frac{\sqrt{1+x}-1}{\sqrt{1+x}+1}\right)\right\} .
\end{aligned}
$$

Upon extracting the mass and wave-function renormalization from the above expression, we recover the result of Ref. [18].

\section{CONCLUSIONS}

We have presented a simple technique for calculating the expansion of lattice integrals around their continuum limit. The method is based on two observations. First, using techniques developed for continuum integrals, it is possible to systematically expand the lattice integrals in a series in the lattice spacing. The expansion is nonanalytic and requires introducing an intermediate regularization to make the expansion of the integrands possible. After the expansion, the original integral is a sum of two distinct contributions. The first one is a set of analytically regularized continuum integrals which encode the small momentum behavior of the original lattice integral and depend in a non-trivial way on the internal momenta and masses. The remaining part is a polynomial in the momenta and masses with massless lattice tadpole-integrals as coefficients. Within a given theory, these tadpole-integrals are process-independent and need to be calculated only once.

We have shown how to efficiently calculate massless lattice tadpoles. Integration-by-parts identities relate different lattice tadpole-integrals. These relations are rather complicated, and their analytic solution is difficult. However, it is not necessary. A robust approach is to use the algorithm suggested in [15] which allows the solution of the recurrence-relations and the reduction of all the lattice tadpole-integrals to a few master integrals to be performed in an automated fashion. We have applied this algorithm to tadpole-integrals in gluodynamics, HQET on the lattice and QCD with Wilson fermions. We 
have illustrated the flexibility of the method by computing a number of two-point functions in lattice perturbation theory such as the gluon and fermion self-energies and the static fermion self-energy in an arbitrary gauge.

In the future, it will be interesting to see if this method can be generalized to higher orders in perturbation theory. It is clear that a similar separation of the integrals into process-dependent soft and universal hard parts is possible; it should also be possible to derive integrationby-parts relations for the hard parts of the lattice integrals. A potential problem could arise from the size and the complexity of the system of equations governing the reduction of the massless tadpole-integrals at the twoloop level. In principle, the size of the system is not very relevant, but for practical reasons, such as available CPU time and memory requirements, it might impose severe constraints.

Although it may sound paradoxical, more precise data from lattice simulations will not eliminate the need for perturbation theory. In many cases the nonperturbative results from simulations need to be matched by equally precise perturbative calculations in lattice regularization in order to become phenomenologically relevant. Using the method discussed in this paper, such calculations are not much more difficult than perturbative calculations in continuum field theory. Hopefully, this simplification will persist at higher orders.

Acknowledgments: We are grateful to Frank Petriello for useful suggestions and to Charalampos Anastasiou for sharing some of his insight in solving recurrence relations by computer algebra. This research was supported by the DOE under grant number DE-AC03-76SF00515.
[1] For an introduction, see H. J. Rothe, "Lattice Gauge Theories: An Introduction," World Sci. Lect. Notes Phys. 59, 1 (1997).

[2] K. Symanzik, Nucl. Phys. B 226, 187 (1983), K. Symanzik, Nucl. Phys. B 226, 205 (1983).

[3] S. Sint and P. Weisz [ALPHA collaboration], Nucl. Phys. B545, 529 (1999).

[4] M. Lüscher and P. Weisz, Phys. Lett. B349, 165 (1995).

[5] M. Lüscher and P. Weisz, Nucl. Phys. B452, 234 (1995).

[6] B. Alles, A. Feo and H. Panagopoulos, Nucl. Phys. B491, 498 (1997).

[7] T. Reisz, Commun. Math. Phys. 116, 81 (1988). T. Reisz, Commun. Math. Phys. 116, 573 (1988).

[8] S. Caracciolo, P. Menotti and A. Pelissetto, Nucl. Phys. B375, 195 (1992).

[9] G. Burgio, S. Caracciolo and A. Pelissetto, Nucl. Phys. B478, 687 (1996).

[10] K. G. Wilson, "Quarks: From Paradox To Myth," in Erice 1975, Proceedings, New Phenomena In Subnuclear Physics, Part A, New York 1977, 13-32.

[11] M. Lüscher and P. Weisz, Nucl. Phys. B445, 429 (1995)

[12] For a review, see V. A. Smirnov, "Applied Asymptotic
Expansions In Momenta And Masses," Berlin, Germany: Springer (2002) 262 p.

[13] F. V. Tkachov, Phys.Lett. B100, 65 (1981);

K. G. Chetyrkin and F. V.Tkachov, Nucl. Phys. B192, 159 (1981).

[14] The numerators occurring in the evaluation of Feynman diagrams involve powers of $\sin \left(k_{\mu}\right)=2 \eta_{\mu} /\left(\eta_{\mu}^{2}+1\right)$ and $\cos \left(k_{\mu}\right)=\left(1-\eta_{\mu}^{2}\right) /\left(1+\eta_{\mu}^{2}\right)$.

[15] S. Laporta, Int. J. Mod. Phys. A15, 5087 (2000).

[16] C. Anastasiou, E. W. Glover and M. E. Tejeda-Yeomans, Nucl. Phys. B629, 255 (2002).

[17] E. Eichten and B. Hill, Phys. Lett. B 234, 511 (1990).

[18] E. Eichten and B. Hill, Phys. Lett. B240, 193 (1990).

[19] We note that the hard master integrals in two dimensions can be evaluated analytically: $H(\{1,1\} ; 1)=-\pi / \delta+$ $3 \pi \ln 2+O(\delta)$ and $2 H(\{1,1\} ; 2)=-\pi / \delta-\pi+3 \pi \ln 2+$ $O(\delta)$.

[20] A. Hasenfratz and P. Hasenfratz, Phys. Lett. B 93, 165 (1980).

[21] H. Kawai, R. Nakayama and K. Seo, Nucl. Phys. B189, 40 (1981). 